\title{
15 Die Tiefe Hirnstimulation
}

Uta Bittner, Christian Lenk, Jan Kassubek und Heiner Fangerau

\section{1 Übersicht über Versuche zur Tiefen Hirnstimulation in den vergangenen 150 Jahren - eine kurze Darstellung des medizin- und kulturgeschichtlichen Hintergrundes}

Jüngere Arbeiten zum Einsatz der Tiefen Hirnstimulation erwecken vielfach den Eindruck, es handele sich bei der therapeutisch intendierten elektrischen Stimulation des menschlichen Gehirns um eine Erfindung der späten 198oerund frühen 199oer-Jahre (Hariz et al. 2010, E1-E2). Eine solche Sichtweise, die vielleicht in Bezug auf aktuelle Techniken und Einsatzbereiche nachvollziehbar erscheinen mag, verstellt nur zu leicht den Blick darauf, dass sich eine mehr als 20o-jährige neurophysiologische Traditionslinie von Experimenten zur elektrischen Erregung des Gehirns zeichnen lässt und dass seit den 193oerJahren die elektrische Stimulation tiefer Hirnstrukturen auch mit der Zielsetzung einer Behandlung z.B. epileptischer und psychischer Störungen durchgeführt wurde (Feindel 1992; Stahnisch 2011).

Zu den ersten Aufsehen erregenden Versuchen, überhaupt die Wirkung elektrischen Stroms auf das menschliche Gehirn zu untersuchen, zählen die Demonstrationen von Giovanni Aldini (1762-1834). In seinem Bestreben, die von Luigi Galvani (1737-1798) beschriebene „tierische Elektrizität“ zu untersuchen und öffentlich vorzuführen, nahm er Stimulationsexperimente an Gehirnen von Ochsen und Hingerichteten vor (Parent 2004). Seine wohl berühm- 
teste Vorführung erfolgte 1803 in London an der Leiche des eben erhängten George Foster. Die Times vom 22. Januar 1803 berichtete von diesem Experiment. In dem Bericht heißt es, dass nach der Applikation des Stroms der Tote nicht nur die Gesichtsmuskeln verzerrt habe, sondern auch ein Auge geöffnet habe, sodass die Umstehenden Sorge gehabt hätten, er würde wieder zum Leben erweckt. In den Versuchen Aldinis erblickte der damalige Berichterstatter auch unter dem Eindruck von Aldinis Aussage, er habe mit Erfolg den galvanischen Strom auch an psychiatrischen Patienten ausprobiert, eine „Entdeckung von großem und vielleicht unvorhersehbarem Nutzen" (The Times 22.01.1803, S. 3). Dieser Prophezeiung zum Trotz wurden Aldinis Studien in der Neurophysiologie zunächst nicht weiter verfolgt. Vielmehr setzte sich die Anschauung durch, dass es sich beim Gehirn um eine unerregbare Masse handele, bis Gustav Fritsch (1838-1927) und Eduard Hitzig (1828-1907) im Jahr 1870 eine vielbeachtete Arbeit „Ueber die elektrische Erregbarkeit des Crosshirns“ veröffentlichten (Fritsch u. Hitzig 1870). Wie viele andere Forscher bemühten sie sich darum, Hirnfunktionen bestimmten Hirnarealen zuzuordnen. Doch anders als ihre Kollegen, die vornehmlich mit Läsionsstudien arbeiteten, nutzten sie die Elektrizität, um beispielsweise zu zeigen, dass bei Versuchstieren (Hunden) die Stimulation des einen anterioren Hirnlappens zu Muskelkontraktionen an der kontralateralen Körperseite führte (Finger 1994).

Nachdem wenig später David Ferrier (1843-1928) epileptische Erscheinungen bei Hunden durch Hirnstimulation ausgelöst hatte, folgte bereits die erste Beschreibung einer elektrischen Reizung am menschlichen Kortex. Robert Bartholow (1831-1904) beschrieb 1874, dass er bei einer sterbenden Patientin durch Elektrostimulation ihrer Hirnrinde Körperbewegungen und Kribbelgefühle in der zum Stimulationsort kontralateralen Seite auslösen konnte. Der Schädel der Patientin war durch ein Basalzellkarzinom erodiert, sodass er mit Elektroden bis zur Hirnrinde gelangen konnte und seine Versuche nach eigenen Angaben ohne Hirnverletzung durchführen konnte. Auch gab er an, die Patientin habe der Durchführung der Versuche zugestimmt. Als die Patientin kurz nach seinen Experimenten starb, wurde jedoch öffentliche Kritik laut, die genau diese Aussagen hinterfragte. Die Patientin war als „geistesschwach“ beschrieben worden, was ihre Einwilligungsfähigkeit in die Versuche fraglich erscheinen ließ, die Zeitgenossen kritisierten, dass die Patientin vermutlich Schmerzen während der Prozedur erlitten hatte und es wurde die Frage gestellt, ob nicht vielleicht Bartholows Versuche den Tod der Patientin beschleunigt hätten (Walker 1957; Morgan 1982).

Neben zahlreichen Lokalisationsexperimenten an Tieren wurden bis in die 1940er-Jahre hinein in der Folge vor allem Versuche zum Einsatz von Elektrostimulation in der Diagnostik und Behandlung von Epilepsien durchgeführt, wobei die Stimulationsverfahren hier und auch in der Psychiatrie als Alternative zu oder Erleichterung von neurochirurgischen Eingriffen gesehen und propagiert wurden (Arends u. Fangerau 2011; Stahnisch 2011). Neurochirurgen 
15.1 Übersicht über Versuche zur Tiefen Hirnstimulation in den vergangenen 150 Jahren eine kurze Darstellung des medizin- und kulturgeschichtlichen Hintergrundes

wie Otfried Foerster (1873-1941) oder Wilder Penfield (1891-1976) nutzten in den 1920er- und 193oer-Jahren Stimulationen dazu, während der Operationen vitales Gewebe von beispielsweise Narbengewebe zu unterscheiden, um so Strukturen, die nicht verletzt werden sollten, möglichst sicher zu erhalten (Schwalb u. Hamani 2008). Mit der Entwicklung stereotaktischer Operationsmethoden durch zum Beispiel Ernest Spiegel (1895-1985) und Henry Wycis (1911-1972) wurden diese Verfahren verbessert und auch tiefe Hirnstrukturen zielgenauer für Elektroden zugänglich. Seitdem gibt es ununterbrochene Forschungslinien zum Beispiel zur Stimulationsfrequenz in unterschiedlichen Einsatzgebieten, zum Einsatz der Tiefen Hirnstimulation bei psychiatrischen Patienten oder zur Veränderung des Verhaltens, zur Behandlung von Schmerz, zur Behandlung von Epilepsien oder zur Behandlung von Bewegungsstörungen (Hariz et al. 2010).

Von besonderer Brisanz erscheinen für die ethische Bewertung des Verfahrens die verschiedenen Versuche, menschliches Verhalten durch Hirnstimulation zu modifizieren oder psychische Erkrankungen zu behandeln (siehe hierzu vertiefend die Kap. 15.2 und 15.7). Bereits im Jahr 1954 fasste der amerikanische Psychiater Robert Heath eine Reihe seiner Studien zur Tiefen Hirnstimulation von psychisch Kranken in seinem Buch „Studies on Schizophrenia“ zusammen (Heath 1954). Bei ca. einhundert Patienten mit unterschiedlichen Diagnosen stimulierte er vor allem die Septumregion, um schizophrene Symptome zu behandeln. Die Rationale seiner Therapien und die zeitgenössischen Reaktionen sind ausführlich von Baumeister beschrieben worden, der am Ende seiner Arbeit zu dem Schluss kommt, dass Heaths Experimente, die er alle mit einem therapeutischen Anspruch begründete, nicht nur von einigen Zeitgenossen kritisiert wurden, sondern wohl auch den ethischen Standards der Zeit nicht genügt hätten (Baumeister 2000). Einen aus heutiger Sicht höchst problematischen Höhepunkt erreichten die Versuche Heaths 1972, als er mit Moan eine Arbeit publizierte, in der er über die Einleitung heterosexuellen Verhaltens bei einem homosexuellen Mann durch Stimulation mit Elektroden an verschiedenen Stellen des limbischen Systems, der Septumregion und des Kortex berichtete. Das „Behandlungsprogramm“ bestand aus passiver (extern gesteuerter) und aus Selbststimulationen, dem Anschauen von Pornofilmen, Masturbation und zuletzt dem Geschlechtsverkehr mit einer Prostituierten. Während einer vorherigen Phase der Selbststimulation musste der Patient gegen seinen Willen vom Auslöser getrennt werden, weil er sich in ein von den Untersuchern für ungut gehaltenes Hochgefühl stimuliert hatte. Eine mögliche Schlussfolgerung ihres Fallberichts sahen Moan und Heath darin, dass es eventuell doch möglich sein müsste, von extern als angemessen erachtetes Verhalten in Menschen hervorzurufen, indem über Lust erzeugende Stimulationen eine positive Verstärkung erwünschten Verhaltens hervorgerufen werden könnte (Moan u. Heath 1972). In eine ähnliche Richtung gingen auch die Überlegungen von José Delgado (1915-2011), der bis heute unter anderem dafür berühmt ist (Horgan 2005), dass er sich mit einem Stier in eine Arena begab, in 
dessen Gehirn Elektroden implantiert worden waren, die Delgado mittels einer Fernsteuerung auslösen konnte. In dem Moment, in dem der Stier auf ihn zuraste, um ihn auf die Hörner zu nehmen, brachte er ihn mittels Fernsteuerung zum Stehen. Auch Delgado hatte sich seit Anfang der 1950er-Jahre bemüht, psychotische Symptome mit Tiefer Hirnstimulation zu beherrschen (Delgado et al. 1952) und kurze Zeit vor Moans und Heaths Ideen zum Einsatz der Tiefen Hirnstimulation zu Erziehungszwecken hatte Delgado in seinem Buch „Physical Control of the Mind: Toward a Psychocivilized Society “ auf Basis eigener Tierversuche ebenfalls Überlegungen dazu angestellt, ob es nicht sinnvoll sei, die Menschen technisch mithilfe von Hirnelektroden zu zivilisierterem Verhalten zu zwingen (Delgado 1969; Fangerau u. Martin 2011).

Es scheint so, als habe sich nach Heaths Experimenten mit dem homosexuellen Mann und den folgenden Debatten die Forschung im Umfeld der Tiefen Hirnstimulation zunächst auf die Stimulation thalamischer Strukturen zur Behandlung von Bewegungsstörungen, Schmerz und Epilepsie konzentriert. Seit den späten 1980er-Jahren stehen der Nucleus subthalamicus, der Globus pallidus und der Nucleus ventralis intermedius des Thalamus und ihre Stimulation zur Behandlung von Symptomen des Morbus Parkinson sowie anderer Bewegungsstörungen (und auch versuchsweise zur Behandlung der Depression) im Vordergrund (Kringelbach et al. 2007). Für andere Indikationsbereiche wie Schmerzsymptome, z.B. der Clusterkopfschmerz, der aufgrund seiner hohen Intensität in pharmakotherapierefraktären Fällen immer wieder zu Suiziden der schmerzgeplagten Patienten führt, steht die Tiefe Hirnstimulation im Bereich des Hypothalamus ebenfalls als Therapieoption zur Verfügung, bei heterogener Datenlage wird sie jedoch nicht flächendeckend angewendet (Magis et al. 2012). Doch spätestens seit der Einsatz der Tiefen Hirnstimulation zur Behandlung von Zwangssymptomen kaum noch außer Frage und der Gedanke an ihren Einsatz zur Behandlung psychiatrischer Symptome wieder im Raum steht, erscheinen die gelegentlich vergessenen Vorläufer eines Einsatzes der Tiefen Hirnstimulation zur Modulation von Psyche und Verhalten für die heutige ethische Debatte bedenkenswert (vgl. auch die ausführlichere Übersicht bei Clausen 2010a). Zumindest erscheint es bemerkenswert, dass diese Traditionslinie entweder gelegentlich in Vergessenheit geraten zu sein scheint oder zu Gunsten der Annahme einer technischen Lösungsmöglichkeit auch für die früheren ethischen Probleme der Tiefen Hirnstimulation (Fangerau 2011) als irrelevant betrachtet wird.

\subsection{Ausprägungen und gängige Formen}

Vor dem Hintergrund der verschiedenen historischen Vorläufer der Stimulation von Hirnarealen ist zu klären, wie es mit der Verbreitung und Zulassung der Tiefen Hirnstimulation (THS; engl.: deep brain stimulation, DBS) in der jüngeren Vergangenheit aussieht. 
Zwei wesentliche Anwendungsgebiete für die THS werden im folgenden Text unterschieden:

- So wird die Tiefe Hirnstimulation zum einen bei der Behandlung von $B e-$ wegungsstörungen (movement disorders) eingesetzt. Dieser Anwendungsbereich ist gut etabliert und zählt die häufigsten Eingriffe (s. Kap. 15.3). Seit dem Jahr 1997 hat die amerikanische Food and Drug Administration (FDA) das Verfahren der Tiefen Hirnstimulation für die Behandlung von Tremor anerkannt; fünf Jahre später erfolgte die Zulassung für Morbus Parkinson (vgl. Bell et al. 2010).

- Mittlerweile wird zum anderen wieder darüber verhandelt, das Indikationsspektrum auszuweiten, etwa auch auf psychiatrische Erkrankungen (zum Beispiel zur Behandlung des Tourette-Syndroms oder von starken Depressionen). 2009 kam die spezielle Zulassung durch die FDA (, humanitarian device exemption“; Fins et al. 2011) für starke Zwangserkrankungen, auch als obsessive compulsive disorders (OCD) bekannt (vgl. Bell et al. 2010). Damit ist auch der zweite Anwendungsbereich eröffnet, in dem derzeit psychiatrische Erkrankungen (wie etwa schwerste Depressionen oder Zwangserkrankungen) behandelt werden, bei denen anderweitige Behandlungsformen keine bzw. kaum Wirkung mehr erzielen. Dieser Anwendungsbereich ist im Vergleich zum Einsatz der THS bei Bewegungsstörungen weit weniger verbreitet und befindet sich größtenteils noch im experimentellen Stadium: „DBS for psychiatric diseases is clinical research on therapeutics" (Schlaepfer et al. 2011, S. 4).

Im Folgenden liegt der Schwerpunkt auf der Darstellung der THS im Bereich der Bewegungsstörungen. Da aber auch die Eingriffe zur Behandlung von psychiatrischen Krankheiten zunehmen und zudem die dort bewusst herbeigeführten Veränderungen von Persönlichkeitsmerkmalen, Verhaltensweisen, kognitiven oder emotionalen Einstellungen in besonderer Weise diskutiert werden, soll auch dieses Einsatzgebiet vorgestellt werden.

Vor der Hirnstimulation steht die Implantation von Stimulationselektroden. Während der eigentlichen neurochirurgischen Operation ist der Patient wach. Das ist möglich, da das Gehirn kein Schmerzempfinden hat. Die Operation wird mit einem stereotaktischen Operationssystem durchgeführt. Nach einer Kurznarkose oder (in der Regel) Lokalanästhesie der Kopfhaut werden die Elektroden in einem stereotaktischen Verfahren eingebracht. Die Platzierung am richtigen Ort erfolgt mit Orientierung an Magnetresonanztomografie (MRT) oder Computertomografie (CT), stereotaktischem Atlas und Signalen der eingeführten Elektrode. Die Elektrode wird mit einem Impulsgeber verbunden, der in einer zweiten Operation z.B. unter der Haut auf dem Brustmuskel implantiert wird. Für weiterführende Details zum stereotaktisch-neurochirurgischen Vorgehen und Operationsverfahren bei der Tiefen Hirnstimulation kann auf die Empfehlungen der Deutschen Arbeitsgemeinschaft Tiefe Hirnstimulation verwiesen werden (vgl. Voges et al. 2009). 
Lyons nennt als Hauptrisiken der Operation u.a. Blutungen, Infektionen, sowie Bruch, Fehlplatzierung oder Verschiebung des Drahtes (vgl. Lyons 2011, S. 662). Er geht von einer Morbiditätsrate von 3-4\% aufgrund der Operation aus, hier existieren jedoch sehr große Unterschiede zwischen z.B. unterschiedlichen Ländern. Hilker et al. berichten u.a. von einer „operationsbedingten Mortalität von 0,4\%“ (Hilker et al. 2009, S. 646).

\subsubsection{Behandlung des Morbus Parkinson}

Gemäß der Darstellung von Lyons (vgl. Lyons 2011, S. 663) richtet sich die Behandlung des Morbus Parkinson mit der Tiefen Hirnstimulation in erster Linie gegen die motorischen „Kardinalsymptome“ der Erkrankung, nämlich Tremor, Bradykinesie (also verlangsamte Bewegungsabläufe) und Rigor(Muskelsteifigkeit). Nachdem die Wirkung der medikamentösen Behandlung im Lauf der Zeit abgenommen hat, kann die Tiefe Hirnstimulation eine zusätzliche gute Langzeitwirkung vorweisen (vgl. exemplarisch Hilker et al. 2009). Nach der Darstellung von Lyons (Lyons 2011) stellen bei der medikamentösen Behandlung auch Arzneimittel-induzierte Bewegungsstörungen ein großes Problem dar, welche unter der THS-Behandlung gemindert werden können. Somit verbessert die THS bei idiopathischem Parkinson-Syndrom neben den motorischen Symptomen auch die Lebensqualität der Patienten, indem Alltagsaktivitäten wieder besser gemeistert werden können (vgl. Schnitzler et al. 2010 oder auch Witt et al. 2008, S. 610). In der Leitlinie der Arbeitsgemeinschaft der Wissenschaftlichen Medizinischen Fachgesellschaften AWMF (S2Leitlinie Neurologie, Parkinson Syndrome) wird die Tiefe Hirnstimulation als

„fester Bestandteil der Therapie und [...] als eine potente Behandlungsmethode der Parkinson-Krankheit im fortgeschrittenen Stadium mit Dopasensitiven Fluktuationen“ (AWMF 2012, Onlineversion, S. 2)

bezeichnet und festgestellt, dass sie in einigen Fällen der medikamentösen Therapie überlegen ist (AWMF 2012, S. 2). Schon 2006 haben Deuschl et al. die Wirkung der THS bei Parkinson untersucht und kamen zu dem Ergebnis, dass THS die Krankheitssymptome stärker verbessern konnte als die alleinige medikamentöse Behandlung (Deuschl et al. 2006b). So berichten Deuschl et al. unter anderem, dass Parkinson-Patienten unter THS im Vergleich zu Patienten, die nur eine medikamentöse Behandlung erhielten, mehr Aktivitäten im Alltag verfolgten, ein höheres emotionales Wohlbefinden (emotional well-being) aufwiesen oder etwa weniger Stigmatisierungserfahrungen erlebten (Deuschl et al. 2006b, S. 904, 907). Die mit THS behandelten Patienten in ihrer Studie hätten „longer periods and better quality of mobility with less dyskinesia“ (Deuschl et al. 2006b, S. 907). Gleichfalls stellten sie fest, dass beispielsweise kognitive Funktionen und der Stimmungszustand unverändert blieben (,[c] ognition, mood, and overall psychiatric functioning were unchanged“; 
Deuschl et al. 2006b, S. 907). Allerdings scheint die Wirkung der THS bei Parkinson-Patienten auch vom Alter der Patienten abhängig zu sein. So schreiben Schnitzler et al.:

„Die Lebensqualität bessert sich signifikant unter der THS, allerdings scheint dieser Effekt bei älteren Patienten geringer zu sein als bei jüngeren." (Schnitzler et al. 2010, S. S39; vgl. auch Hilker et al. 2009, S. 646).

In den „Empfehlungen der Deutschen Arbeitsgemeinschaft Tiefe Hirnstimulation“ zum THS-Einsatz bei idiopathischem Parkinson-Syndrom wird darauf hingewiesen, dass die „Auswahl geeigneter Patienten für die tiefe Hirnstimulation [...] einen höchst individuellen Entscheidungsfindungsprozess" darstellt (Hilker et al. 2009, S. 646). In einer solchen Auswahl werden, wie Hilker et al. schreiben, zum Beispiel der „Zustand“ der Betroffenen und potenziellen THS-Patienten sowie ihre „krankheitsbezogenen Einschränkungen der Lebensqualität“" genauso berücksichtigt wie ihr „berufliche[r] und soziale[r] Status“ (Hilker et al. 2009, S. 646). Und es gilt zudem: „Eine manifeste Demenz stellt eine Kontraindikation zur THS dar“ (Hilker et al. 2009, S. 649). Weitere Kontraindikation für eine THS sind etwa

\begin{abstract}
„biologisches [wohlbemerkt nicht kalendarisches; Anmerkung der Autoren] Lebensalter über 75 Jahre, schwere internistische Allgemeinerkrankungen, maligne Grunderkrankungen mit eingeschränkter Lebenserwartung, chronische Therapie mit Immunsuppressiva, chronische, nicht zu unterbrechende Antikoagulation, ausgeprägte innere und/oder äußere Hirnatrophie, relevante strukturelle Hirnläsionen, [...], schweres frontal-dysexekutives Syndrom, manifeste Psychose, klinisch relevante Störungen von Affekt (Depression, Manie) und/oder Verhalten(Substanzmissbrauch, Dopamindysregulationssyndrom) und/oder Persönlichkeit, latente oder manifeste Suizidalität (Suizidgedanken, Suizidhandlungen in der Vorgeschichte)“ (Hilker et al. 2009, S. 650, Aufzählungszeichen im Original wurden nicht übernommen).
\end{abstract}

\title{
15.2.2 Behandlung des essentiellen Tremor
}

Neben dem Tremor, der als Symptom von Morbus Parkinson auftritt, wird auch der „essentielle Tremor“ mittels THS behandelt. Der essentielle Tremor, eine der häufigsten neurologischen Erkrankungen, betrifft am häufigsten die Hände, kann jedoch auch Kopf, Stimme sowie die unteren Extremitäten beeinträchtigen (Lyons 2011, S. 664; vgl. auch Sixel-Döring et al. 2009). Besonders die Einschränkung der Funktion der Hände führt dazu, dass alltägliche Verrichtungen wie essen, trinken oder schreiben beeinträchtigt werden (vgl. Deuschl et al. 2011, S. 149). Vor allem beim Hand-Tremor scheint eine THS genau dann eine attraktive Option, wenn eine medikamentöse Behandlung keine adäquate Symptomlinderung bewirkt (vgl. Deuschl et al. 2011, S. 154; siehe auch Sixel-Döring et al. 2009, S. 662). Als Kontraindikationen für eine THS bei essentiellem Tremor werden angeführt: 
„Demenz, signifikante psychiatrische Komorbidität wie schwere Depression, Psychose, Substanzmissbrauch oder relevante Persönlichkeitsstörung, höhergradige Hirnatrophie und/oder Leukenzephalopathie, allgemeine chirurgische Kontraindikationen wie schlechter Allgemeinzustand, Immunsuppression, Antikoagulation, Komorbidität mit erheblich reduzierter Lebenserwartung. "(Sixel-Döring et al. 2009, S. 662, Aufzählungszeichen im Original wurden nicht übernommen).

Der essentielle Tremor tritt häufiger mit steigendem Alter auf und folgt in betroffenen Familien einem dominanten Vererbungsmuster (vgl. Lyons 2011, S. 664). Die Publikationen mit erfolgreichen Berichten zu der Behandlungsform mit THS gehen von einer Kontrolle des Tremors bei 70\% bis 90\% der behandelten Patienten aus (vgl. Lyons 2011, S. 664). Allerdings nimmt die Wirkung der Stimulation im Laufe der Zeit üblicherweise ab, beispielsweise als Ausdruck der Progression der Erkrankung, sodass dann eine höhere Stimulationsstärke gewählt werden muss (vgl. Sixel-Döring et al. 2009, S. 665). Insgesamt scheint die Datenlage zur Diagnose und den diversen Behandlungsformen des essentiellen Tremors noch verbesserungsbedürftig, sodass Deuschl et al. in ihrer Untersuchung u.a. zu dem Ergebnis kommen:

"Safety and efficacy of the different DBS targets(Vim nucleus, prelemniscal radiation, zona incerta, and subthalamic nucleus) are unknown and need to be investigated further." (Deuschl et al. 2011, S. 157)

Auch fordern Deuschl et al. für zukünftige Forschungen zu wirkungsvollen Behandlungsformen des essentiellen Tremors eine stärkere Einbindung und Berücksichtigung der Lebensqualität als Bewertungsmaßstab (vgl. Deuschl et al. 2011, S. 157).

\subsubsection{Behandlung der Dystonie}

Zur Behandlung der Dystonie (einer Gruppe von Bewegungsstörungen, bei denen unwillkürliche Muskelanspannungen zu Verkrampfungen und Fehlhaltungen führen) hat die Deutsche Arbeitsgemeinschaft Tiefe Hirnstimulation Empfehlungen zum Einsatz der THS ausgesprochen (vgl. Schrader et al. 2009). Hier wird zunächst darauf hingewiesen, dass „[d]ie Behandlung von an Dystonie erkrankten Patienten [...] mangels gut wirksamer Medikamente erfahrungsgemäß schwierig“ sei (Schrader et al. 2009, S. 656; vgl. auch Kupsch et al. 2006, S. 1979). Alternative therapeutische Maßnahmen, die in der Vergangenheit durchgeführt wurden, wie etwa „läsionelle neurochirurgische Verfahren (Thalamotomien und Pallidotomien)" (Schrader et al. 2009, S. 656) zeigten zwar, so Schrader et al., in Bezug auf die Dystonie Effekte, seien aber auch „mit zum Teil intolerablen Nebenwirkungen“ verbunden (ebd.). Die THS könne hier „als sicheres und wirksames Verfahren“ (ebd.) neue Behandlungswege eröffnen, wobei die Autoren der Empfehlungen auch darauf hinweisen, dass es vielfältige Formen von Dystonien gebe und nach Schrader et al. „nur für die Behandlung von generalisierter Dystonie bzw. Rumpfdystonie ausrei- 
chende Daten“ vorlägen, die „eine leitliniengerechte Empfehlung rechtfertigen“ (für beide Zitate: Schrader et al. 2009, S. 656). Kupsch et al. kamen in ihrer Studie zu dem Ergebnis, dass die Medikamentendosis für die Behandlung der Dystonie nach sechsmonatiger THS bei einigen Patienten um 32,1\% reduziert werden konnte (vgl. Kupsch et al. 2006, S. 1987). Außerdem wurde durch die THS der Depressionsgrad gesenkt, ohne dabei den „mental or cognitive status" zu verändern (Kupsch et al. 2006, S. 1987). Nach sechsmonatiger THS hätten sich bei den Dystonie-Patienten die Bewegungsmöglichkeiten, der Behinderungsgrad und die Lebensqualität (Kupsch et al. 2006, S. 1978, 1983) deutlich verbessert. Einzig beim Sprechen und Schlucken seien keine wesentlichen Verbesserungen festgestellt worden (vgl. Kupsch et al. 2006, S. 1978, 1983). In den USA ist die THS bei Dystonie eingeschränkt zugelassen (vgl. Schrader et al. 2009, S. 656). Dennoch gilt es noch immer zu beachten, dass es

„bei der Dystonie bislang keine Kriterien [gibt; ergänzt durch die Autoren], anhand derer Art und Ausmaßdes Stimulationseffektes individuell und verlässlich vorhergesagt werden können“"(Schrader et al. 2009, S. 658).

Auch spricht die Deutsche Arbeitsgemeinschaft Tiefe Hirnstimulation keine Empfehlung zum besten Zeitpunkt einer THS-Operation aus, weil hier die Datenlage für noch nicht ausreichend erachtet werde (vgl. Schrader et al. 2009, S. 658).

\subsubsection{Einsatzgebiete bei psychiatrischen Erkrankungen}

Neuropsychiatrische Erkrankungsbilder wurden bisher viel seltener mittels THS behandelt und in weniger Studien untersucht als der Morbus Parkinson, der essentielle Tremor oder auch die Dystonien (vgl. auch Kap. 15.3). Im Folgenden soll kurz auf die verschiedenen Einsatzgebiete von THS bei psychiatrischen Erkrankungen eingegangen werden, auch wenn die Fallzahlen hierzu (noch) sehr niedrig sind (vgl. Kap. 15.3).

Vielfach ist noch ungeklärt, welche Gehirnregionen (targets) bei welchen Grunderkrankungen stimuliert werden sollen, um den besten Effekt zu erzielen (vgl. z.B. Schlaepfer et al. 2011, S. 2-3). Lyons etwa weist darauf hin, dass beim Einsatz von THS im Fall von Zwangsstörungen (OCD) auch schwerwiegende Nebenwirkungen wie Suizidgedanken oder Hypomanie auftreten können (Lyons 2011, S. 667-668). Eine Sonderstellung nimmt das Tourette-Syndrom ein, da es klinisch sowohl durch eine Bewegungsstörungskomponente (motorische Tics) als auch durch ein Spektrum von vielfältigen neuropsychiatrischen Symptomen gekennzeichnet ist (vgl. Robertson 2000). Aber auch hier geht Lyons (Lyons 2011, S. 667) davon aus, dass der THS-Behandlungsansatz noch nicht vollständig ausgereift sei:

"Although DBS surgery is considered to have a relatively low risk of morbidity and mortality, the optimal target has yet to be determined. Systematic study of this condition and the optimal target is necessary." (Lyons 2011, S. 667) 
Müller-Vahl et al. urteilen in ihren Empfehlungen zur THS beim TouretteSyndrom ähnlich und fordern weitere Forschung in Form von randomisierten, doppel-blind kontrollierten Studien (vgl. Müller-Vahl et al. 2011, S. 215). Ebenso ist Lyons vorsichtig beim Einsatz der THS zur Therapie der Epilepsie, so stellt er fest, dass auch in diesem Bereich zum jetzigen Zeitpunkt „Placebo-Effekte nicht vollständig ausgeschlossen werden“ könnten (Lyons 2011, S. 668; Übersetzung durch die Autoren).

Die Beurteilungen der THS bei psychiatrischen Erkrankungen fallen oft noch sehr unterschiedlich aus. So wird - im Gegensatz zu Lyons - bei Kuhn et al. die Tiefe Hirnstimulation bei psychiatrischen Erkrankungen (Zwangsstörungen, Tourette-Syndrom, Depressionen) insgesamt positiver eingeschätzt, sodass in der Zusammenfassung sogar von „,beeindruckende[n] Therapieergebnisse[n]" (vgl. Kuhn et al. 2010, S. 105) gesprochen wird (im Resümee der Publikation allerdings vergleichsweise vorsichtiger, vgl. Kuhn et al. 2010, S. 112). Das Ziehen belastbarer Schlussfolgerungen im Bereich psychiatrischer Erkrankungen dürfte stark von der Tatsache erschwert werden, dass Ergebnisse größerer Studien in diesem Bereich nicht zur Verfügung stehen. So legen Kuhn und Kollegen selbst nach einer systematischen Literaturrecherche dar, dass es sich bei den Publikationen zur THS-Therapie der Zwangsstörung in vielen Fällen lediglich um Fallbeschreibungen handelt und zum Zeitpunkt ihrer $\mathrm{Pu}$ blikation nur „fünf Arbeitsgruppen ermittelt werden [konnten; ergänzt durch die Autoren], die über mehr als drei Patienten berichteten, bei denen Zwangsstörungen mit THS behandelt wurden“ (Kuhn et al. 2010, S. 106). Fins et al. fordern in ihrem kritischen Artikel von 2011 dementsprechend das Durchführen einer systematischen Studie im Bereich der THS-Therapie der Zwangsstörung und das Vermeiden weiterer einzelner „Heilversuche“ (Fins et al. 2011).

Zusammenfassend gilt so in Bezug auf die Anwendung der Tiefen Hirnstimulation bei psychiatrischen Erkrankungen festzuhalten, dass letztlich die Tiefe Hirnstimulation im Bereich der psychiatrischen Erkrankungen im Gegensatz $\mathrm{zu}$ den vorher genannten Indikationen bei Bewegungsstörungen zum gegenwärtigen Zeitpunkt als wenig etabliert eingestuft werden muss. Die zum Teil verschiedenen Einschätzungen in den konsultierten Übersichtsartikeln von Lyons (Lyons 2011) sowie Kuhn et al. (Kuhn et al. 2010) zeigen aber auch die Wichtigkeit eines angemessenen Maßstabes und machen den Ruf nach qualifizierten Forschungsdaten zur Beurteilung der Tiefen Hirnstimulation verständlich, um unrealistische Hoffnungen bei der Behandlung psychiatrischer Erkrankungen zu vermeiden und realistische Erwartungshorizonte abzustecken.

\subsection{Häufigkeiten}

Die Angabe von Häufigkeiten zur Anzahl der Indikationen und den konkreten Anwendungszahlen der THS stellt ein schwieriges Unterfangen dar. So ent- 
stehen immer neue THS-Zentren, die Eingriffe vornehmen. Im Folgenden können daher für die Eingriffszahlen im Zeitverlauf nur Näherungswerte angeführt werden.

Um dennoch ein wenig Orientierung zu bieten, sind in Tabelle 1 für das Jahr 2008 die bis dahin mit Tiefer Hirnstimulation behandelten Patienten nach Indikationen geordnet aufgeführt. Bei diesen Zahlen handelt es sich um eine Schätzung der Autoren aus der funktionalen Neurochirurgie des John Radcliffe Hospital, Oxford. Hierbei ist zu beachten, dass diese Zahlen aus dem Jahr 2008 stammen und seitdem relevant angestiegen sind.

\section{Tab. 1 Indikationen für Tiefe Hirnstimulation und ungefähre Zahl der weltweit behandelten Patienten nach Pereira et al. (2008, S. 21)}

\begin{tabular}{l|c}
\hline Indikation & Anzahl der weltweit behandelten Patienten \\
\hline Morbus Parkinson & 40.000 \\
\hline chronische Schmerzen & 2.000 \\
\hline Tremor (außerhalb der Diagnose Morb. Park.) & 1.000 \\
\hline Dystonie & 500 \\
\hline Cluster Kopfschmerzen & 100 \\
\hline Epilepsie & 50 \\
\hline Tourette-Syndrom & 100 \\
\hline Obsessive-compulsive disorder, Zwangsstörung & 100 \\
\hline Depression & 50 \\
\hline
\end{tabular}

Die Zahlen der Statistik stimmen jedoch gut mit den zitierten Studien aus dem vorangegangenen Abschnitt überein, denen zufolge die nach Morbus Parkinson, Schmerzen, Tremor und Dystonien weiteren Indikationen für THS (Cluster Kopfschmerzen, Epilepsie, Tourette-Syndrom, Zwangsstörung, Depression) auch im internationalen Überblick nur in sehr kleinen Zahlen gestellt wurden.

So ist festzuhalten: Die größte Bedeutung und weiteste Verbreitung findet das THS-Verfahren bei Bewegungsstörungen. Hier stellt vor allem die Behandlung von idiopathischem Parkinson-Syndrom, essentiellem Tremor und Dystonien mittels Tiefer Hirnstimulation mittlerweile eine etablierte und gut erforschte Behandlungsform dar (auch wenn weiterhin noch großer Forschungsbedarf besteht). Dies spiegelt sich auch in den Empfehlungen der Deutschen Arbeitsgemeinschaft Tiefe Hirnstimulation wieder, die selbige zu essentiellem Tremor, Parkinson und Dystonie veröffentlicht hat (vgl. Sixel-Döring et al. 2009, Hilker et al. 2009, Schrader et al. 2009). Der Bereich der THS bei psychiatrischen Erkrankungen ist im Vergleich wesentlich kleiner. Hier besteht noch erheblicher und elementarer Forschungsbedarf, um die jeweiligen Wirksamkeiten der THS näher zu bestimmen und zu validieren. 


\subsection{Psychische Aspekte: Motive der Patienten}

Die Motive der Patienten, sich einer Operation und anschließender Stimulation zu unterziehen, sind je nach Krankheitsbild unterschiedlich; im Allgemeinen kann jedoch davon ausgegangen werden, dass eine Linderung des Leidens an den Krankheitssymptomen als dominantes Motiv für die Entscheidung zur Tiefen Hirnstimulation im Vordergrund steht. Denn die THS wird in einer Abwägung von Risiko (auch intra- und perioperativ) und voraussichtlichem Nutzen vor allem solchen Patienten angeboten und bei diesen durchgeführt, bei denen pharmakologische oder andere Behandlungswege keinen nachhaltigen Erfolg mehr versprechen (vgl. Clausen 201ob; exemplarisch für den essentiellen Tremor vgl. Sixel-Döring et al. 2009). Sie befinden sich in der Regel in den Stadien der schweren Krankheitsausprägung; Hilker et al. etwa schreiben in ihren Empfehlungen zum Einsatz der THS bei idiopathischem Parkinson-Syndrom zwar, dass „[g]eeignete Patienten für eine THS [...] eine Mindesterkrankungsdauer von 5 Jahren aufweisen“ (Hilker et al. 2009, S. 646) sollten - allerdings scheint die tatsächliche Erkrankungsdauer bis zu einem THS-Eingriff wesentlich länger zu sein. So berichten Schnitzler et al., dass THS-Eingriffe bei Parkinson ,erst nach einer mittleren Krankheitsdauer von etwa 14 Jahren durchgeführt" würden (Schnitzler et al. 2010, S. S37, S38). Doch es gibt auch schon erste Untersuchungen und begonnene Studien, die aufzeigen, dass ein früherer Einsatz der THS durchaus auch erfolgversprechend sein könnte. In ihrer Untersuchung zu einem früheren Einsatz von THS bei Parkinson kommen Schnitzler et al. in ihrer Zusammenfassung zu dem Schluss:

„Nach klinischen Pilotdaten von Patienten, die eine THS bereits nach durchschnittlich 7-jährigem Krankheitsverlauf im Stadium beginnender Wirkfluktuationen erhielten, könnte ein Einsatz in diesem Krankheitsstadium die motorischen Funktionen und die Lebensqualität frühzeitiger bessern, die sozialen Folgen der Erkrankung wie Arbeitsplatzverlust und gesellschaftliche Isolation mildern und den therapeutischen Nutzen auf einen längeren Zeitraum ausdehnen." (Schnitzler et al. 2010, S. S37)

Noch stehen die abschließenden Ergebnisse der in diesem Zusammenhang sehr wesentlichen deutsch-französischen EARLYSTIM-Studie aus, in der untersucht wird, inwiefern die THS bei deutlich jüngeren Parkinson-Patienten mit einer wesentlich kürzeren Erkrankungszeit wirkt (zum Forschungsdesign der EARLYSTIM-Studie vgl. Deuschl et al. 2012).

Insgesamt wird deutlich, dass der jeweilige Leidensdruck bei den Patienten sehr hoch ist und daraus mitunter große Hoffnungen auf die Wirkung der THS erwachsen. Doch diese Hoffnung auf eine erfolgreiche Hilfe durch das THSVerfahren muss deshalb nicht schon berechtigt oder angemessen sein: So berichten beispielsweise Bell und Kollegen von Patienten mit zu hohen oder unrealistischen Erwartungen (Bell et al. 2010, S. 115). Allerdings ist zur Studie von Bell et al. ergänzend hinzuzufügen, dass sie in semi-strukturierten Inter- 
views einzig die Perspektive von healthcare providers erhoben haben, und somit diese ihre Sicht der THS-Patienten schildern und nicht die Patienten selber. Dennoch erscheinen diese Einschätzungen interessante Diskussionsfelder zu eröffnen. So berichten Bell et al., dass healthcare providers der Ansicht sind, dass nicht selten auch die vielfältige positive mediale Berichterstattung (,the media's tendency to report best-case-scenario outcomes“, Bell et al. 2010, S. 117) über sehr gut gelaufene THS-Eingriffe zu überbordenden Erwartungen bei den Patienten beitrüge (vgl. Bell et al. 2010, S. 116f.). Hier müsse entsprechend aufgeklärt werden. Auch scheint es notwendig, immer wieder darauf hinzuweisen, dass die THS einzig die Symptome behandele, aber keine Heilung der Krankheiten insgesamt bewirke. Das heißt, dass die Patienten und Angehörigen akzeptieren müssen, dass es auch mit Tiefer Hirnstimulation kein Leben ohne die Erkrankung geben wird. Diese Akzeptanz zu erreichen ist jedoch mitunter schwer. Es ist offensichtlich, dass es folglich einer individuell auf den Patienten ausgerichteten realistischen Aufklärung vonseiten des Arztes bedarf, um ein sich ansonsten einstellendes „Unzufriedenheitsdilemma“ (Krug 2010, S. 134) zu vermeiden. Neben diesem prä-operativen Erwartungsmanagement besteht außerdem auch nach der Operation und während der Stimulation (und Stimulationseinstellung) die Notwendigkeit eines ausführlichen post-operativen Managements (vgl. Deuschl et al. 2006a; Sixel-Döring et al. 2009). Denn auch wenn beispielsweise die Parkinsonmedikation zum Teil nach der Operation deutlich reduziert werden kann (vgl. Perozzo et al. 2001, S. 81), wird nach der Operation in der Regel nicht vollständig auf die Medikamenteneinnahme verzichtet werden können.

Allerdings gibt es auch durchaus Patienten (und Angehörige), die mit realistischen Erwartungen der THS begegnen. Beide Gruppen - Patienten wie ihre Angehörigen - sind nach ausführlichen Aufklärungsgesprächen durch die interdisziplinär zusammengesetzten Ärzteteams auf die Möglichkeiten der THS-Behandlung vorzubereiten (zur Forderung multidisziplinärer Teams siehe z.B. Bell et al. 2010) und können sodann einschätzen, welche Wirkungsweise die THS in Aussicht stellt. Bei solchermaßen „aufgeklärten“ Patienten und ihren Angehörigen sind auch post-operative Enttäuschungen über zu hohe und daher notwendigerweise fehlgeschlagene Erwartungen vermutlich weniger anzutreffen (vgl. Clausen 201ob).

\subsection{Umgang im medizinischen Alltag}

Ärzte können mit zu großen Erwartungshaltungen bzw. möglichen Enttäuschungen in der Weise umgehen, als dass sie die THS-Patienten immer wieder darauf hinweisen, welche Einschränkungen vor dem THS-Eingriff bestanden (s.o.). Wenn durch die Tiefe Hirnstimulation einige, aber nicht alle Symptome gelindert werden konnten, so sollte diese Verbesserung den THS-Patienten deutlich gemacht werden, damit sie sehen, worin die Besserung besteht. Al- 
lerdings ist auch zu beachten, dass mitunter Verbesserungen der Symptome wie etwa die Reduzierung der Tics beim Tourette-Syndrom nicht unbedingt zu einer verbesserten Lebensqualität bei den Betroffenen führen (vgl. MüllerVahl et al. 2011, S. 212); es kann also durchaus Unterschiede in der objektiven und subjektiven Bewertung der THS-Wirkung geben (vgl. Krug 2010).

Um die Patienten vor überzogenen Erwartungen und daraus resultierenden hohen Enttäuschungsgraden zu bewahren, scheint es ebenfalls wichtig, nicht nur die individuelle Erwartungshaltung der THS-Patienten genau zu eruieren, sondern ebenfalls die Einstellungen und Perspektiven der Angehörigen abzufragen und im Gespräch vor und nach einem THS-Eingriff zu thematisieren (vgl. Hilker et al. 2009, S. 646; Perozzo et al. 2001, S. 82). Denn nach Operationen können sich durchaus vormalige soziale (Abhängigkeits-)Verhältnisse verändern (vgl. Fogel u. Moringlane 2004, S. 418f.). Dies kann auch mitunter zu Verschiebungen und Umgewichtungen bzw. Neu-Definitionen innerhalb der familiären Strukturen führen, indem die Partner und Angehörigen des THS-Patienten sich auf die neue Unabhängigkeit ihres Angehörigen lernen müssen einzustellen und mit dieser neuen Situation umzugehen (vgl. Fogel $\mathrm{u}$. Moringlane 2004). Gelingt diese Umstellung und Neu-Justierung innerhalb familiärer, freundschaftlicher oder nachbarschaftlicher Strukturen nicht, kann dies zu (innerfamiliären) Brüchen, Scheidungen, Streit oder anderweitigen Konflikten und Dissensen führen (vgl. Fogel u. Moringlane 2004).

Auch hierauf hat das behandelnde Ärzteteam im Rahmen der Aufklärung und Vorbereitung des Eingriffs ausführlich und intensiv hinzuweisen und zu informieren. So fordern Schüpbach et al. eine multidisziplinäre, vor allem auch psychosoziale Betreuung von Patienten vor und nach der THS-Operation (vgl. Schüpbach et al. 2006). Dennoch schafft es die beste Aufklärung in manchen Fällen nicht, Patienten vor überzogenen Erwartungen zu schützen (vgl. Bell et al. 2010, S. 119). Volkmann et al. weisen zudem darauf hin, dass prä-operative Dispositionen und Auffälligkeiten von ärztlicher Seite genauestens erhoben werden sollten, was sich jedoch auch als problematisch erweisen kann, da die Patienten mitunter beschämt sind über ihre auffälligen Verhaltensweisen in ihrem Leben und nicht freiwillig darüber berichten wollen - u.a. aus Angst vor einem Ausschluss von der THS-Operation (vgl. Volkmann et al. 2010, S. 494).

Gleichfalls ist im klinischen Kontext auch zu beachten, ob und inwiefern die Patienten in der Lage sind, ihre informierte Zustimmung und Einwilligung in die Behandlung und in Forschungsstudien zu geben. Da die THS in vielen Bereichen noch verstärkt untersucht und mit Forschungsstudien begleitet werden soll (s.o.), gilt es, auch die Problematik der „decisional capacity and therapeutic misconception“ (Fisher et al. 2012, S. 69) zu berücksichtigen, wie dies etwa Fisher und Kollegen am Beispiel der THS bei Depression untersucht haben (Fisher et al. 2012). 
Es wird auch von medizinischer Seite empfohlen, die Aufklärung, Information und Vorauswahl von potenziellen THS-Patienten in multidisziplinären Teams durchzuführen (Bell et al. 2010, S. 121). Für die Selektion von Patienten für eine THS gibt es mitunter bereits ausführliche Vorgaben und Empfehlungen, die je nach Krankheitsbild/Indikation auch spezifisch ausgearbeitet sind (siehe zum Beispiel für das idiopathische Parkinson-Syndrom: Hilker et al. 2009 oder für das Tourette-Syndrom: Müller-Vahl et al. 2011). Gleichzeitig sollte sich das Ärzteteam auch der mitunter starken Wirkung medialer Darstellungen von THS bewusst sein und dieses Wissen in das aufklärende und informierende, individuelle Arzt-Patienten-Gespräch genauso integrieren wie die Perspektiven von Angehörigen der Patienten (Bell et al. 2010, S. 121, 123).

\subsection{Nebenwirkungen und Gefahren}

Wie schon in Kapitel 15.2 angeführt, können die Nebenwirkungen und Wechselwirkungen beim Einsatz der Tiefen Hirnstimulation zum einen bei der Durchführung des operativen Eingriffes auftreten (siehe die Morbiditätsraten und Mortalitätsraten bei der Operation). Zudem sind in Anlehnung an Kuhn et al. 2010 noch weitere Nebenwirkungsbereiche zu benennen: So sind einerseits Probleme auf der technischen Ebene - zum Beispiel des THS-Gerätes (wie zum Beispiel „Kabelbruch, Fehlfunktion des Neurostimulators“; Kuhn et al. 2010, S. 109) denkbar -, und es kann andererseits zu verschiedenen „Nebenwirkungen durch die eigentliche Stimulation“ (Kuhn et al. 2010, S. 109; Kursivsetzung durch die Autoren) kommen.

In der Literatur wird von verschiedenen Nebenwirkungen berichtet, die durch die Tiefe Hirnstimulation selbst evoziert werden (können). Im Folgenden werden einige dieser potenziellen unerwünschten Effekte kurz in exemplarischer Form dargestellt.

Die Nebenwirkungen hängen zum einen von dem Zielgebiet der Stimulation $\mathrm{ab}$, aber auch von der Grunderkrankung des Patienten oder seiner allgemeinen Grunddisposition sowie von anderen Faktoren wie etwa der Einstellung der Stimulationsparameter. In verschiedenen Studien sind die Nebenwirkungen und ihre Häufigkeiten evaluiert worden (siehe hierzu u.a. die Übersicht von Volkmann et al. $2010 \mathrm{zu}$ den neuropsychiatrischen Nebenwirkungen der THS bei Parkinson-Patienten; vgl. auch Schüpbach et al. 2006 sowie Clausen 201ob). So können nach THS-Eingriffen bei Parkinson-Patienten u.a. unerwünschte Nebenwirkungen wie zum Beispiel Gewichtszunahme, Sprachstörungen, Muskelkontraktionen oder Verhaltensveränderungen auftreten (für eine umfassende Darstellung vgl. Deuschl et al. 2006a, S. S219), deren Behandlung das Arzt- und Pflegeteam prä- und postoperativ berücksichtigen muss. Je nach 
Stimulationsgebiet, Medikamentenreduktion oder Grundverhaltensmuster der mit THS behandelten Parkinson-Patienten sind - wie Volkmann et al. berichten - verschiedene neuropsychiatrische Nebenwirkungen wie zum Beispiel Manie, Depression, Probleme bei Entscheidungsprozessen, Apathie, Euphorie, Suizid bzw. versuchter Suizid, Hypersexualität, Angst und Panikattacken oder auch impulse control disorders beobachtet worden - wobei zum Teil noch ungeklärt ist, inwiefern diese Effekte auf die Tiefe Hirnstimulation, die Grunderkrankung oder die Medikation zurückzuführen sind (vgl. Volkmann et al. 2010). Im Hinblick hierauf zeigten sich auch an kleinen Fallserien mit unterschiedlichen Untersuchungsmethoden immer wieder Hinweise auf Verhaltensänderungen nach THS, die allerdings mehrere Einflussfaktoren aufwiesen (vgl. Lulé et al. 2012). Daher variieren die Forschungsergebnisse auch mitunter: So kommen Witt et al. bei ihrer Untersuchung der neuropsychiatrischen Nebenwirkungen der THS bei der Behandlung von Morbus Parkinson zu dem Ergebnis, dass die THS nicht die allgemeinen kognitiven Leistungen und Affektivität beeinflusst („DBS [...] does not reduce overall cognition or affectivity, although there is a selective decrease in frontal cognitive functions and an improvement in anxiety in patients after the treatment", Witt et al. 2008, S. 605). Bemerkenswert ist, dass die neuropsychiatrischen Nebenwirkungen vor allem in den ersten post-operativen Monaten aufzutreten scheinen (vgl. Volkmann et al. 2010, S. 495).

Auf langfristige negative Effekte weisen Deuschl et al. hin, wenn sie beschreiben, dass etwa Infektionen oder ein nachlassender Stimulationseffekt, Schmerzen oder Unwohlsein (,discomfort“) (Deuschl et al. 2006a, S. S219) auftreten können. Daher plädieren Deuschl und Kollegen für ein umfassendes post-operatives Management dieser Nebenwirkungen und Effekte.

Beim THS-Einsatz bei Dystonie-Patienten scheint zudem die Häufigkeit von Hardware-Defekten (wie etwa Kabel- oder Elektrodenbrüche) höher zu sein als bei THS-Patienten mit einer anderen Grunderkrankung (vgl. Schrader et al. 2009, S. 660 sowie vgl. Kupsch et al. 2006, S. 1988). Zudem tritt nach Schrader et al. speziell bei THS-Patienten mit Dystonie die Wirkung der Stimulation verzögert - nach 6 Monaten - ein, manchmal auch erst nach 12 Monaten (vgl. Schrader et al. 2009, S. 660 sowie Kupsch et al. 2006, S. 1988). Speziell bei Dystonie-Patienten empfiehlt die Deutsche Arbeitsgruppe Tiefe Hirnstimulation auch eine „intensive physiotherapeutische Mitbetreuung“ (Schrader et al. 2009, S. 660) in den ersten Monaten nach Stimulationsbeginn, weil die Patienten vielfach unter den Folgen der jahrelangen Dystonie „sekundäre Veränderungen (Muskelverkürzungen, unphysiologische kompensatorische Bewegungsabläufe)“ ausgebildet hätten, die behandelt werden müssten (Schrader et al. 2009, S. 660).

Auch von möglichen Nebenwirkungen in Bezug auf das Wohlbefinden bzw. die Zufriedenheit mit dem äußeren Erscheinungsbild der THS-Patienten, das durch die technischen Implantate Veränderungen erfährt, wird berichtet. So 
stört THS-Patienten mitunter die Sichtbarkeit des unter die Haut implantierten Stimulationsgeräts oder des unter der Haut entlanggeführten Kabels (vgl. Fogel u. Moringlane 2004).

Nebenwirkungen können nur den Patienten selber betreffen (sein körperliches und psychisches Wohlbefinden). Aber auch (mitunter gravierende) Effekte auf das soziale Zusammenleben (in Familie, Beruf, usw.) und auf etablierte Rollenverständnisse werden erwähnt (vgl. etwa Fogel u. Moringlane 2004, S. 418f.). Hier sind als Folgen der Stimulation soziale bzw. inter-subjektive Effekte beobachtet worden (s.o.). So haben beispielsweise Perozzo et al. in ihrer Studie 15 Patienten (9 Männer, 6 Frauen), die an Morbus Parkinson erkrankt sind und mit Tiefer Hirnstimulation behandelt wurden, begleitet und die Veränderungen und Komplikationen in ihrem Sozialverhalten (mit ihren Angehörigen) beobachtet. Perozzo und Kollegen beobachteten kurz nach der THS-Operation: „the great improvement in PD motor symptoms led to a corresponding improvement of mood for both the patients and their caregivers" (Perozzo et al. 2001, S. 81) und „patients and families began to plan a series of projects for the future. " (Perozzo et al. 2001, S. 81). Jedoch mussten in den Monaten nach der Operation die Stimulationsparameter und die Medikamenteneinstellung justiert werden; obwohl die Patienten, wie Perozzo et al. schreiben, von der Notwendigkeit dieser Re-Justierungen wussten, waren doch etwa 70\% der Patienten ängstlich, dass sich ihr Zustand wieder auf das Niveau des prä-operativen Zustands verschlechtern würde (vgl. Perozzo et al. 2001, S. 82). Neben diesen Ängsten befürchteten die Patienten auch einen Verlust an Kontrolle und die Angehörigen eine erneute Autonomieeinschränkung:

"Since the stimulation parameters can be varied only by physicians, several patients felt a sense of impotence and passivity regarding their clinical condition, and were afraid of not being able to control the new situation. The caregivers were worried to lose the just acquired autonomy, demanding a re-establishment of social and relative role." (Perozzo et al. 2001, S. 82)

Auch wenn bei neuen Stimulatorgenerationen Funktionen wie die Wiederaufladbarkeit durch den Patienten vorhanden sind und einzelne Variationen der Parameter in einem vorgegebenen Rahmen vom Patienten bzw. Angehörigen selbstständig vorgenommen werden können, scheint dies keine Lösung für diesen subjektiven Kontrollverlust darzustellen.

Als weiteren psychologischen Aspekt, der auf die Gestaltung intersubjektiver Beziehungen rückwirkt, führen Perozzo et al. in ihrer Studie an, dass THSPatienten mitunter auch Probleme hätten, auf die mit der Erkrankung einhergehenden Vorteile wie etwa der bevorzugten Behandlung durch größere Aufmerksamkeit oder der geringeren Verantwortungsübernahme in Bezug auf Alltagsverrichtungen zu verzichten („had difficulty to give up the benefits deriving from the illness condition, such as more attention from the relatives or the irresponsibility toward everyday matters.” Perozzo et al. 2001, S. 82). 
Dieses komplexe Geflecht an Interdependenzen und Dynamiken in sozialen Strukturen von Nahbeziehungen nach einem THS-Eingriff ist nicht zu unterschätzen (vgl. Schüpbach et al. 2006). Perozzo et al. raten daher zu psychologischer Begleitung: „to allow the patients to gradually adapt to the post-operative period without trauma." (Perozzo et al. 2001, S. 82). Und explizit in Bezug auf die Angehörigen fordern Perozzo und Kollegen: „caregivers ought to continue to support patients and to understand that psychological changes are slower than the motor improvement deriving from the surgery. "(Perozzo et al. 2001, S. 82).

Auch im Kontext der THS bei Dystonie wird auf soziale Herausforderungen im Kontext des THS-Einsatzes hingewiesen:

„Besonders bei Kindern und Jugendlichen kann die psychosoziale Nachbetreuung relevant sein, da hier häufig durch die jahrelange, dystoniebedingte Invalidität soziale Folgeschäden (z.B. durch fehlenden Schulabschluss, fehlende Sozialisierung) eingetreten sind." (Schrader et al. 2009, S. 660).

Auch für THS-Patienten mit Tourette-Syndrom empfehlen Müller-Vahl et al. das Angebot einer psychotherapeutischen Begleitung (vgl. Müller-Vahl et al. 2011, S. 213).

\subsection{Gesetzliche Regelungen und ethische Aspekte}

Unter Juristen und Ärzten besteht heutzutage Einigkeit, dass der behandelnde Arzt den Patienten vollumfänglich über alle Risiken und möglichen Nebenwirkungen des THS-Einsatzes zu informieren hat. Erst wenn der Patient wohlinformiert (in Kenntnis der relevanten Daten und Fakten sowie Risiken) in die Behandlung mittels THS eingewilligt hat (,informed consent“, informierte Einwilligung), darf der Arzt die Tiefe Hirnstimulation durchführen und die Sonden operativ in das Gehirn einsetzen. Das Aufklärungsgespräch hat dabei den allgemeinen ärztlichen Richtlinien der Bundesärztekammer zu entsprechen (vgl. BÄK 199o). Dennoch ist nicht auszuschließen, dass unterschwellige Hoffnungen die Erwartungshaltung in Bezug auf das Ergebnis der THS beeinflussen - auch wenn die Aufklärung vollumfänglich erfolgte (s.o.). Diese Faktoren sind aber de facto in einem klinischen Setting kaum vollständig zu lösen. Darüber hinaus ist im Hinblick auf rechtliche Aspekte beim THS-Eingriff auch die Einwilligungsfähigkeit des Patienten zu bedenken, wenn es sich beispielsweise um Patienten mit schweren psychiatrischen Erkrankungen oder um minderjährige Patienten handelt. So wird zum Beispiel die THS auch bei Kindern und Jugendlichen bei Dystonie eingesetzt (vgl. Schrader et al. 2009). Allerdings wird die THS nicht bei allen Erkrankungen gleichermaßen auch bei Jugendlichen oder Kindern genutzt: So wird beispielsweise bei dem Tourette-Syndrome generell davon abgeraten, die THS schon im Kindes- oder Jugendalter anzuwenden, da die Tics sich durchaus noch im Zeitverlauf ver- 
ändern und abklingen können; die potenziellen THS-Patienten mit TouretteSyndrom sollten daher mindestens 18 bzw. 25 Jahre alt sein, so der bisherige Kenntnisstand (vgl. Müller-Vahl et al. 2011, S. 209, 213f.).

Haftungsrechtlich ist die Frage interessant, wer für Ausfälle, Defekte oder Fehlfunktionen seitens des Stimulators eigentlich verantwortlich ist. So sind Szenarien denkbar, in denen das Stimulationsgerät ausfällt und der THS-Patient beispielsweise einen Unfall aufgrund des technischen Defekts verursacht. Weiterhin sind Fälle denkbar, in denen THS-Patienten das Steuerungsgerät nicht sachgemäß bedienen (vgl. auch die Ausführungen bei Foegel u. Moringlane $2004 \mathrm{zu}$ möglichen Alltagsproblemen mit dem Stimulationsgerät).

Ethisch ist das Verfahren der Tiefen Hirnstimulation durchaus noch in Teilaspekten umstritten, obgleich es als Behandlungsverfahren für Bewegungsstörungen im medizinischen Klinikalltag schon etabliert ist. Vor allem der verstärkte, zum Teil noch experimentelle Einsatz der THS bei psychiatrischen Erkrankungen wird diskutiert (vgl. Clausen 2010b). Zudem wird im Kontext um die Legitimität einer Erweiterung des Indikationsspektrums auch angemerkt, dass mittels der THS ganz gezielt und aktiv Einfluss auf Stimmungen sowie auf emotionale, kognitive oder motivationale Aspekte genommen werden kann (vgl. exemplarisch Clausen 2010b). Ob und inwiefern diese Einflussnahme auf die psychische Struktur von Personen wünschenswert sein kann oder nicht, wird aus medizinethischer Perspektive durchaus kontrovers diskutiert. Denn gerade bei psychiatrischen Indikationen wie starker Depression oder starken Zwangserkrankungen scheint es explizites Ziel des therapeutischen Eingriffs per THS zu sein, Verhaltensveränderungen oder Änderungen der emotionalen Grundzustände zu evozieren. Hier stellen sich dann u.a. ethische Fragen nach der Authentizität von durch Hirnschrittmacher erzeugten Verhaltensweisen, Dispositionen, Emotionen und Gefühlen. Die Beantwortung der Frage nach der „Echtheit“ bzw. Urheberschaft ist medizinethisch von großer Bedeutung, da sie mögliche Gefahren und subtile Wirkungen der Tiefen Hirnstimulation in den Blick nimmt. Dabei ist die Debatte - wie oben geschildert - nicht neu, sondern bereits zu Beginn der 1970er-Jahre geführt worden.

Unumstritten scheint der Vorteil zu sein, dass es sich bei der Tiefen Hirnstimulation um ein zumindest in Teilen reversibles Verfahren handelt, das heißt, die Stimulation kann sofort abgeschaltet werden, und die Elektroden können zudem auch wieder aus dem Gehirn entfernt werden. Diese Möglichkeit der sofortigen An- und Abschaltung der Stimulation wird als deutlicher Vorteil gegenüber anderen neurochirurgischen Verfahren erachtet (vgl. Kuhn et al. 2010, S. 106).

Vor der Entscheidung der Anwendung der Tiefen Hirnstimulation werden oftmals die vier medizinethischen Prinzipien - Wohltun, Nicht-Schaden, Respekt vor der Autonomie, Gerechtigkeit (vgl. Beauchamp u. Childress 2009)- herangezogen (Arends u. Fangerau 2011). Neben diesen zentralen bioethischen Prinzipien gilt es aber auch, aus ethischer Perspektive weitere zentrale Aspekte 
wie Entfremdungsphänomene (aufgrund der Technik im Gehirn; vgl. Schüpbach et al. 2006), die Entwicklung neuer sozialer Interaktionsmuster (vgl. Perozzo et al. 2001; Schüpbach et al. 2006), die Bedeutung einer zunehmenden Technisierung des Gehirns (vgl. Hildt u. Engels 2009) bzw. allgemein des menschlichen Körpers und daraus resultierende Wahrnehmungsverschiebungen usw. bei einer ethischen, sozialen und rechtlichen Evaluation der Tiefen Hirnstimulation zu berücksichtigen.

Weiterhin wird das Verfahren der Tiefen Hirnstimulation auch zum Anlass genommen, um über Enhancement-Möglichkeiten nachzusinnen. Erste Anzeichen, dass eine solche Übersteigerung mittels Tiefer Hirnstimulation möglich sein könnte, zeigen Berichte über Formen von z.B. „Gedächtnis-Enhancement" - als Nebeneffekte der Stimulation (vgl. Hamani et al. 2008; siehe auch Bezug bei Clausen 2010b). Auch wenn derzeit im medizinischen Kontext die Tiefe Hirnstimulation rein zu therapeutischen Zwecken eingesetzt wird, wird unter einem erweiterten Blickwinkel über mögliche Zukunftsszenarien, Zielsetzungen und Motive der Einsatzerweiterung für die THS nachgedacht (vgl. Clausen 2010b, S. 1159).

Um weitere, detaillierte Erkenntnisse über die Wirkungsweise der Tiefen Hirnstimulation zu erlangen, scheint auch eine stärkere Berücksichtigung (auto-) biografisch-narrativer Berichte von THS-Patienten und ihren Angehörigen einen (qualitativen) Forschungsansatz zu offerieren (vgl. exemplarisch Schüpbach et al. 2006; Müller et al. 2010; Krug et al. 2010).

\subsection{Fazit für den Umgang im klinischen Alltag}

Insgesamt ist festzuhalten, dass die Behandlung von Bewegungsstörungen wie etwa dem idiopathischen Parkinson-Syndrom oder dem essentiellen Tremor mit dem Verfahren der Tiefen Hirnstimulation eine wichtige Option für die Symptomlinderung und Lebensqualitätssteigerung der betroffenen Patienten darstellt. Doch trotz beeindruckender Erfolge der Tiefen Hirnstimulation sind stets auch die Risiken und Grenzen des Verfahrens zu berücksichtigen. Die behandelten Erkrankungen werden durch die THS nicht geheilt, sondern es erfolgt eine mehr oder weniger effektive Symptomlinderung (wobei die Krankheitssymptome im Zeitverlauf trotz Stimulation wieder stärker werden können und auch die Krankheit selbst fortschreitet). Diese Hilfe in Form von reduzierter Symptomatik kann für die betroffenen Patienten durchaus von gravierender Bedeutung sein, können sie durch eine erfolgreiche THS-Behandlung beispielsweise wieder am Alltagsleben partizipieren, sich selbstbestimmt fortbewegen, in den Beruf zurückkehren und Abhängigkeitsverhältnisse, die durch die fortschreitende Erkrankung entstehen, teilweise abbauen. Bisher macht die Behandlung von Bewegungsstörungen den größten Einsatzbereich der Tiefen Hirnstimulation aus. Hier sind die Wirkungen und Nebenwirkungen zum Teil erforscht und bekannt (gilt vor allem für THS beim idiopathischen Parkinson- 
Syndrom, bei essentiellem Tremor und Dystonien; gleichwohl werden auch hier weiterführende Untersuchungen gefordert, um die Datenlage zu verbessern, vgl. z.B. Kupsch et al. 2006). In dem Einsatzbereich bei psychiatrischen Erkrankungen ist die Datenlage - wie geschildert - wesentlich unausgereifter, die Fallzahlen sind sehr viel geringer. Hier besteht noch großer Forschungs- und Validierungsbedarf zur Wirksamkeit der THS. Abschließend ist der Forderung von Kuhn et al. zuzustimmen, dass die THS „grundsätzlich, aber insbesondere bei psychiatrischen Erkrankungen, von einem laufenden kritisch-ethischen Diskurs begleitet werden“" (Kuhn et al. 2010, S. 112) sollte - dies gilt insbesondere sowohl vor dem Hintergrund der Erfahrungen, die in den vergangenen 150 Jahren mit Versuchen zur THS gemacht wurden und die Erwartungen und Ängste in Bezug auf das Verfahren präfiguriert haben, als auch vor dem Hintergrund der zunehmenden Ausweitung des Indikationsspektrums.

\section{Literatur}

Arends M, Fangerau H (2011) Deep Brain Stimulation in Psychiatric Disorders. In: Fangerau H, Fegert I, Trapp T (Hrsg.) Implanted Minds. The Neuroethics of Intracerebral Stem Cell Transplantation and Deep Brain Stimulation. 137-150. Transcript Bielefeld

AWMF (2012) S2-Leitlinie Neurologie, Parkinson Syndrome - Diagnostik und Therapie. (Autoren: Eggert K, Oertel W, Reichmann H, Arnold G, Baas H, Berg D, Deuschl G, Dodel R, Gasser T, Gerlach M, Höglinger G, Jost W, Mehdorn M, Müngersdorf M, Poewe W, Reuther P, Riederer P, Schulz I, Spieker S, Storch A, Tatsch K, Trenkwalder C, Waldvogel D). Online unter: http://www.awmf.org/uploads/tx_szleitlinien/030-010l_S2k_ Parkinson_Syndreome_Diagnostik_Therapie_2012-09.pdf (abgerufen am 02.10.2013)

BÄK Bundesärztekammer (1990) Empfehlungen zur Patientenaufklärung. Dt Ärzteblatt 87(16), 807-809

Baumeister AA (2000) The Tulane Electrical Brain Stimulation Program a historical case study in medical ethics. I Hist Neurosci 9(3), 262-278

Beauchamp TL, Childress JF (2009) Principles of Biomedical Ethics. Oxford University Press Oxford

Bell E, Maxwell B, McAndrews MP, Sadikot A, Racine E (2010) Hope and Patients' Expectations in Deep Brain Stimulation: Healthcare Providers' Perspectives and Approaches. The Journal of Clinical Ethics 21(2), 112-124

Clausen I (2010a) Technik im Gehirn: Ethische, theoretische und historische Aspekte moderner Neurotechnologie. Deutscher Ärzte-Verlag Köln

Clausen J (2010b) Ethical brain stimulation - neuroethics of deep brain stimulation in research and clinical practice. European Journal of Neuroscience 32, 1152-1162

Delgado I (1969) Physical Control of the Mind: Toward a Psychocivilized Society. Harper \& Row New York

Delgado I, Hamlin H, Chapman WP (1952) Technique of intracranial electrode implacement for recording and stimulation and its possible therapeutic value in psychotic patients. Confin Neurol 12(5-6), 315-319

Deuschl G, Herzog I, Kleiner-Fisman G, Kubu C, Lozano AM, Lyons KE, Rodriguez-Oroz MC, Tamma F, Tröster Al, Vitek JL, Volkmann J, Voon V (2006a) Deep brain stimulation. Postoperative issues. Movement Disorders 21(14), S219-S237

Deuschl G, Schade-Brittinger C, Krack P, Volkmann I, Schäfer H, Bötzel K, Daniels C, Deutschländer A, Dillmann U, Eisner W, Gruber D, Hamel W, Herzog I, Hilker R, Klebe S, Kloss M, Koy I, Krause M, Kupsch A, Lorenz D, Lorenzl S, Mehdorn HM, Moringlane IR, Oertel W, Pinsker MO, Reichmann H, Reuss A, Schneider GH, Schnitzler A, Steude U, Sturm V, Timmermann L, Tronnier V, Trottenberg T, Wojtecki L, Wolf E, Poewe W, Voges I, German Parkinson Study Group, Neurostimulation Section (2006b) A Randomized Trial of Deep-Brain Stimulation for Parkinson's Disease. N Engl I Med 355(9), 896-908 [Erratum in: N Engl I Med 355(12), 1289]

Deuschl G, Schüpbach M, Knudsen K, Pinsker M0, Cornu P, Rau J, Agid Y, Schade-Brittinger C (2012) Stimulation of the subthalamic nucleus at an earlier disease stage of Parkinson's disease: Concept and standards of the EARLYSTIM-study. Parkinsonism Relat Disord 28, 1-6 
Deuschl G, Raethjen I, Hellriegel H, Elble R (2011) Treatment of patients with essential tremor. Lancet Neurol 10(2), 148-161

Fangerau H (2011) Brain, Mind and Regenerative Medicine: Ethical Uncertainties and the Paradox of their Technical Fix. In: Fangerau H, Fegert I, Trapp T (Hrsg.) Implanted Minds: The Neuroethics of Intracerebral Stem Cell Transplantation and Deep Brain Stimulation. 15-30. Transcript Bielefeld

Fangerau H, Martin M (2011) Kontrolle des Lebendigen: Medizin und Menschenmaschinen. In: Stederoth D, Hoyer T (Hrsg.) Der Mensch in der Medizin. Kulturen und Konzepte. 161-182. Verlag Karl Alber Freiburg

Feindel W (1992) Brain physiology at the Montreal Neurological Institute: some historical highlights. Journal of Clinical Neurophysiology 9, 176-194

Finger S (1994) Origins of neuroscience. A history of explorations into brain function. Oxford University Press New York

Fins II, Mayberg HS, Nuttin B, Kubu CS, Galert T, Sturm V, Stoppenbrink K, Merkel R, Schlaepfer TE (2011) Misuse Of The FDA's Humanitarian Device Exemption In Deep Brain Stimulation For Obsessive-Compulsive Disorder. Health Aff 30(2), 302-311

Fisher CE, Dunn LB, Christopher PP, Holtzheimer PE, Leykin Y, Mayberg HS, Lisanby SH, Appelbaum PS (2012) The ethics of research on deep brain stimulation for depression: decisional capacity and therapeutic misconception. Ann N Y AcadSci 1265(1), 69-79

Fogel W, Moringlane IR (2004) Tiefe Hirnstimulation - Alltagsprobleme und ihre Bewältigung. In: Krauss JK, Volkmann I (Hrsg.) Tiefe Hirnstimulation. 414-419. Steinkopff Darmstadt

Fritsch G, Hitzig E (1870) Ueber die elektrische Erregbarkeit des Grosshirns. Archiv für Anatomie, Physiologie und wissenschaftliche Medicin 300-332

Hamani C, McAndrews MP, Cohn M, Oh M, Zumsteg D, Shapiro CM, Wennberg RA, Lozano AM (2008) Memory Enhancement Induced by Hypothalamic/Fornix Deep Brain Stimulation. Ann Neurol 63, 119-123

Hariz MI, Blomstedt P, Zrinzo L (2010) Deep brain stimulation between 1947 and 1987: the untold story. Neurosurg Focus 29(2), E1

Heath RG (1954) Studies in schizophrenia: a multidisciplinary approach to mind-brain relationships. Harvard Univ. Press Cambridge Mass

Hildt E, Engels EM (Hrsg.) (2009) Implantate im Gehirn: Eine Einführung. In: Hildt E, Engels EM (Hrsg.) Der implantierte Mensch. Therapie und Enhancement im Gehirn. 11-19. Alber Freiburg

Hilker R, Benecke R, Deuschl G, Fogel W, Kupsch A, Schrader C, Sixel-Döring F, Timmermann L, Volkmann J, Lange M (2009) Tiefe Hirnstimulation bei idiopathischem Parkinson-Syndrom. Empfehlungen der Deutschen Arbeitsgemeinschaft Tiefe Hirnstimulation. Nervenarzt 80, 646-655

Horgan I (2005) The Forgotten Era of Brain Chips. Scientific American Oct 293, 66-73

Kringelbach ML, Jenkinson N, Owen SL, Aziz TZ (2007). Translational principles of deep brain stimulation. NatRevNeurosci 8(8), 623-635

Krug H (2010) Das Unzufriedenheitsdilemma: ein scheinbares Paradox und seine ethischen Implikationen. In: Inthorn I (Hrsg.) Richtlinien, Ethikstandards und kritisches Korrektiv: Eine Topographie ethischen Nachdenkens im Kontext der Medizin. 134-141. Edition Ruprecht Göttingen

Krug H, Müller O, Bittner U (2010) Technisierung des Ich? Überlegungen zu einer ethischen Beurteilung der tiefen Hirnstimulation unter Verwendung von Patienten-Narrationen. Fortschritte der Neurologie - Psychiatrie 78(11), 644-651

Kuhn J, Gründler TO|, Lenartz D, Sturm V, Klosterkötter |, Huff W (2010) Tiefe Hirnstimulation bei psychiatrischen Erkrankungen. Dt. Ärzteblatt 107(7), 105-113

Kupsch A, Benecke R, Müller I, Trottenberg T, Schneider GH, Poewe W, Eisner W, Wolters A, Müller JU, Deuschl G, Pinsker MO, Skogseid IM, Roeste GK, Vollmer-Haase I, Brentrup A, Krause M, Tronnier V, Schnitzler A, Voges I, Nikkhah G, Vesper I, Naumann M, Volkmann |; Deep-Brain Stimulation for Dystonia Study Group (2006) Pallidal Deep-Brain Stimulation in Primary Generalized or Segmental Dystonia. N Engl J Med 355(19), 1978-1990

Lulé D, Heimrath J, Pinkhardt EH, Ludolph AC, Uttner I, Kassubek I (2012) Deep brain stimulation and behavioural changes: is comedication the most important factor? Neurodegener Dis $9(1), 18-24$

Lyons MK (2011) Deep Brain Stimulation: Current and Future Clinical Applications. Mayo Clin Proceedings 86(7), $662-672$ 
Magis D, Jensen R, Schoenen I (2012) Neurostimulation therapies for primary headache disorders: present and future. Curr Opin Neurol 25(3), 269-276

Moan C, Heath RG (1972) Septal Stimulation for the Initiation of Heterosexual Behavior in a Homosexual Male. Journal of Behavioural Therapy and Experimental Psychiatry 3, 23-30

Morgan JP (1982) The first reported case of electrical stimulation of the human brain. I Hist Med Allied Sci 37(1), 51-64

Müller 0, Bittner U, Krug H (2010) Narrative Identität bei Therapie mit Hirnschrittmacher. Zur Integration von Patienten-Selbstbeschreibungen in die ethische Bewertung der tiefen Hirnstimulation. Ethik in der Medizin 22(4), 303-315

Müller-Vahl KR, Cath DC, Cavanna AE, Dehning S, Porta M, Robertson MM, Visser-Vandewalle V; ESSTS Guidelines Group (2011) European clinical guidelines for Tourette syndrome and other tic disorders. Part IV: deep brain stimulation. Eur Child Adolesc Psychiatry 20(4), 209-217

Parent A (2004) Giovanni Aldini: from animal electricity to human brain stimulation. Can I Neurol Sci 31(4), 576-584

Pereira E, Nandi D, Aziz T (2008) Deep Brain Stimulation: an underused panacea? Advances in Clinical Neuroscience and Rehabilitation 8, 20-24

Perozzo P, Rizzone M, Bergamasco B, Castelli L, Lanotte M, Tavalla A, Torre E, Lopiano L (2001) Deep brain stimulation of subthalamic nucleus: behavioural modifications and familiar relations. Neurological Science 22, 81-82

Robertson MM (2000) Tourette syndrome, associated conditions and the complexities of treatment. Brain 123 Pt 3, 425-462

Schlaepfer TE, Bewernick B, Kayser S, Lenz D (2011) Modulating affect, cognition, and behavior - prospects of deep brain stimulation for treatment-resistant psychiatric disorders. Frontiers in integrative neuroscience 5, article 29, 1-6

Schnitzler A, Fuchs G, Baas H, Dillmann U, Hilker R, Oechsner M (2010) Brauchen wir die frühzeitige tiefe Hirnstimulation beim Morbus Parkinson? Early Deep Brain Stimulation for Parkinson's Disease? Fortschr Neurol Psychiatr 78, Suppl 1, S37-40

Schrader C, Benecke R, Deuschl G, Hilker R, Kupsch A, Lange M, Sixel-Döring F, Timmermann L, Volkmann I, Fogel W, Deutsche Arbeitsgemeinschaft Tiefe Hirnstimulation (2009) Tiefe Hirnstimulation bei Dystonie. Empfehlungen der Deutschen Arbeitsgemeinschaft Tiefe Hirnstimulation. Nervenarzt 80(6), 656-661

Schüpbach M, Gargiulo M, Welter ML, Mallet L, Béhar C, Houeto IL, Maltête D, Mesnage V, Agid Y (2006) Neurosurgery in Parkinson disease: A distressed mind in a repaired body? Neurology 66(12), 1811-1816

Schwalb JM, Hamani C (2008) The history and future of deep brain stimulation. Neurotherapeutics 5(1), 3-13

Sixel-Döring F, Benecke R, Fogel W, Hilker R, Kupsch A, Lange M, Schrader C, Timmermann L, Volkmann I, Deuschl G; Deutsche Arbeitsgemeinschaft Tiefe Hirnstimulation (2009) Tiefe Hirnstimulation bei essenziellem Tremor. Empfehlungen der Deutschen Arbeitsgemeinschaft Tiefe Hirnstimulation. Nervenarzt 80(6), $662-665$

Stahnisch F (2011) The Essential Tension: On Ethical and Historical Conundrums in the Trajectories of Deep Brain Stimulation. In: Fangerau H, Fegert I, Trapp T (Hrsg.) Implanted Minds: The Neuroethics of Intracerebral Stem Cell Transplantation and Deep Brain Stimulation. 151-181. Transcript Bielefeld

Voges J, Kiening K, Krauss JK, Nikkhah G, Vesper J; Deutsche Arbeitsgemeinschaft Tiefe Hirnstimulation (2009) Neurochirurgische Standards bei tiefer Hirnstimulation. Empfehlungen der Deutschen Arbeitsgemeinschaft Tiefe Hirnstimulation. Nervenarzt 80(6), 666-672

Volkmann I, Daniels C, Witt K (2010) Neuropsychiatric effects of subthalamic neurostimulation in Parkinson disease. Nat Rev Neurol 6(9), 487-498

Walker AE (1957) Stimulation and ablation; their role in the history of cerebral physiology. J Neurophysiol 20(4), 435-449

Witt K, Daniels C, Reiff J, Krack P, Volkmann I, Pinsker MO, Krause M, Tronnier V, Kloss M, Schnitzler A, Wojtecki L, Bötzel K, Danek A, Hilker R, Sturm V, Kupsch A, Karner E, Deuschl G (2008) Neuropsychological and psychiatric changes after deep brain stimulation for Parkinson's disease: a randomised, multicentre study. Lancet Neurol 7(7), 605-614 